\title{
Brainstem motor neuron dysmorphology and excitatory/inhibitory imbalance in an animal model of autism
}

\author{
H. Alhelo, R.J. Kulesza Jr. \\ Department of Anatomy, Lake Erie College of Osteopathic Medicine, Erie, PA, United States \\ [Received: 6 November 2020; Accepted: 1 December 2020; Early publication date: 28 September 2021]
}

\begin{abstract}
Background: Autism spectrum disorder (ASD) is a developmental disorder associated with in utero exposure to the antiepileptic valproic acid (VPA) in humans, and similar exposure serves as a validated animal model. Animals exposed to VPA in utero have a number of structural, function and behavioural deficits associated with ASD. Furthermore, VPA-exposed animals have shorter body lengths, lower body and brain weights. This difference in body weight may result from impaired caloric intake due to impaired oropharyngeal function.

Materials and methods: Specifically, it is hypothesized that in utero VPA exposure results in fewer lower motor neurons associated with feeding behaviours, that surviving neurons will exhibit dysmorphology and altered balance of excitatory and inhibitory inputs. Further, it is hypothesized that VPA exposure will result in altered oropharyngeal musculature that will impact skull morphology.

Results: These hypotheses were investigated using quantitative morphometrics and immunofluorescence.
\end{abstract}

Conclusions: Results support dysmorphology and excitatorylinhibitory imbalance and these alterations may contribute to dysphagia and poor weight gain in VPA-exposed animals. (Folia Morphol 2022; 81, 4: 863-873)

Key words: trigeminal, vagus, swallowing

\section{INTRODUCTION}

Autism spectrum disorder (ASD) is a neurodevelopmental condition characterised by impairment in communicative, behavioural, and social domains [1, 3]. ASD is diagnosed at a rate of 1 in 5 children in the United States and 1 in 160 children globally [4, 6, 9, 38]. Furthermore, there is a strong predilection for males as ASD affects approximately 4 males for every 1 female [14, 37].

Valproic acid (VPA) is an antiepileptic drug prescribed for seizures, migraines and bipolar disorder, but its use is advised against during pregnancy be- cause of elevated risk of an ASD diagnosis in offspring $[10,26,39]$. VPA exposure in humans and animal models is known to have widespread impact on the offspring with reported changes in the nervous, cardiovascular and skeletal systems $[8,28]$. Specifically, in utero VPA exposure is associated with neural tube defects, facial deformities, delayed skeletal ossification and ASD. Consistent with this effect on the nervous system in humans, in utero VPA exposure is a validated animal model of ASD $[5,7]$. In fact, VPA-exposed animals have fewer neurons in their auditory brainstem, hyperactive responses to

Address for correspondence: R.J. Kulesza Jr., PhD, Department of Anatomy, Lake Erie College of Osteopathic Medicine, 1858 West Grandview Blvd, Erie, PA 16504, 814-866-8423, United States, e-mail: rkulesza@lecom.edu

This article is available in open access under Creative Common Attribution-Non-Commercial-No Derivatives 4.0 International (CC BY-NC-ND 4.0) license, allowing to download articles and share them with others as long as they credit the authors and the publisher, but without permission to change them in any way or use them commercially. 
pure tone stimuli and reduced axonal projections to the midbrain and thalamus $[12,22,24,25,41,42]$. VPA-exposed animals have evidence of dysfunction within the auditory cortex $[2,13,17]$; they are ataxic and have reduced expression of the calcium binding protein calbindin throughout the brainstem and cerebellum [23, 25, 41]. Consistent with use as a model of ASD, VPA-exposed animals demonstrate a number of ASD-related behavioural disturbances $[27,36]$. Furthermore, VPA-exposed animals demonstrate abnormal body size and weight. Specifically, before weaning, VPA-exposed rats weigh the same as control animals. However, at weaning (postnatal day 21), VPA-exposed animals weigh significantly less, and this difference persists until at least P50 $[23,25]$. At P50, VPA-exposed animals have smaller brain weights [25]. Based on these observations, it is hypothesized that changes in body and brain weight after VPA exposure are attributable to alterations in central control of oropharyngeal musculature. This hypothesis is consistent with previous observations of loss of brainstem lower motor neurons controlling oropharyngeal musculature [30]. This hypothesis was investigated by studying the number and morphology of brainstem lower motor neurons (LMNs) in the trigeminal motor nucleus (TMN), the facial nucleus (FN), the nucleus ambiguous (NA) and the hypoglossal nucleus (HGN). Immunofluorescence was utilised to study the balance of excitatory and inhibitory input to these neurons and osteological measurements were obtained from skull and jaw preparations to study bony features and landmarks.

\section{MATERIALS AND METHODS}

\section{Animals}

All animal handling procedures were reviewed and approved by the Lake Erie College of Osteopathic Medicine Institutional Animal Care and Use Committee (protocol 18-04). Sprague-Dawley rats were bred in a controlled laboratory environment, with free access to food and water under a 12-hour light/dark cycle. Animals underwent timed breeding and recovery of a vaginal plug was designated as embryonic day 0 (E0). On the morning of E6, the pregnant females were placed in an exposure cage for 20 minutes to acclimate to the new environment. On the evenings of E6 to E11, chow was removed from the animal's home cage. On the mornings (08:00) of E7-12 animals were fed a meal of $3.1 \mathrm{~g}$ of peanut butter. On E10 and E12, animals in the VPA exposure group were fed $3.1 \mathrm{~g}$ of peanut butter mixed with $800 \mathrm{mg} / \mathrm{kg}$ VPA. After the animal finished the meal, they were placed back in their home cage with chow. This dosage and exposure paradigm for VPA was chosen to more closely model oral-administration in humans (cf. intraperitoneal injection [30]). This exposure results in consistent litter sizes (10-14 pups; 4-6 male pups) with only occasional litter resorption [22-25, $41,42]$. VPA-exposed and control damns were permitted to deliver pups without interference. There was no notable difference in the size of the litters or the male/female ratio between control and VPA-exposed damns (litters ranged between 10 and 14 pups with 3-5 males). One litter from a VPA-exposed mother appeared to be resorbed at some point after the E12 exposure. On P21 animal were weaned and only male pups were included in the study as gender-specific effects of VPA have been reported [32]. Previous work has established that VPA-exposed animals have poor weight gain after P21, lower body and brain weights at P28 and P50 [23, 25, 41]. Food and water ingestion was not recorded for the mothers or their offspring.

\section{Perfusions and brainstem dissection and sectioning}

On P28, male rats were over-dosed with isoflurane (4\% in $\mathrm{O}_{2}$ at $1.2 \mathrm{~L} / \mathrm{min}$ ) and perfused through the ascending aorta with normal saline followed by $4 \%$ paraformaldehyde in $0.1 \mathrm{M}$ phosphate buffer $(\mathrm{pH}$ 7.2; PFA-PB). The brain was dissected from the skull, trimmed to a block including the brainstem; the right side was marked with a register pin and postfixed in $4 \%$ PFA-PB. Brainstems were sectioned on a freezing stage microtome at a thickness of $50 \mu \mathrm{m}$. Sections were collected in 3 wells. Sections in the first well were used for Giemsa staining (below). Sections in the second well were saved for immunolabelling. Sections from the third well were archived in fixative.

\section{Giemsa staining and morphology}

Sections from well one were collected in rostro-caudal order and mounted on slides from alcohol gelatin on glass slides. Sections were air-dried overnight, and then stained for Nissl substance using Giemsa and sealed under coverslips with Permount (Thermo Fisher Scientific). Giemsa-stained sections were examined using a light microscope under low magnification to locate the TMN, FN, NA, and HGN, and determine their caudal to rostral boundaries on both right and left sides of the brainstem. Cell bodies 
were traced using a $60 \times$ objective and analysed using FIJI [31]. Cell body shape was classified using objective morphological criteria. Estimates of neuronal number were performed in 8 control and 8 VPA-exposed animals as previously described [25, 41, 42].

\section{Immunohistochemistry}

Free-floating sections from well two were collected from 4 control and 4 VPA-exposed animals and washed in $\mathrm{PB}$ three times then blocked in a solution of $\mathrm{PB}, 5 \%$ Triton $\mathrm{X}$ and normal horse serum (NHS). The sections were then transferred to the primary solution composed of PB and NHS, along with rabbit anti-SLC32A1 (VGAT) and mouse anti-VGLUT antibodies that target VGAT and VGLUT, respectively, and incubated on a haler at room temperature overnight. The sections were washed in PB 3 times then transferred to the secondary solution containing goat anti-rabbit 594 and Biotin horse anti-mouse antibodies for 12 hours, followed by streptavidin 488 in PB overnight. The sections were washed three times in $P B$, incubated for 20 minutes in a 1:100 solution of Neurotrace Blue (NTB) 435 in PB, rinsed in PB, mounted on glass slides and sealed under coverslips with Entellan (Sigma-Aldrich). Images of the nuclei were collected from a Leica TCS SP5 confocal microscope. At each region of interest, 3 images were collected: 1 of VGLUT labelling, 1 of VGAT and 1 of NTB. These images were pseudocoloured and overlaid in FIJI. In these composite images the number of perisomatic VGAT and VGLUT immunoreactive (IR) puncta were counted.

\section{Skull dissection}

For study of skull specimens, P28 rats were anesthetised using isoflurane as described above and euthanized by decapitation. Soft tissue was carefully dissected away from the skull and the skull was placed in boiling water until thoroughly cleaned of tissue. The skull and both sides of the mandible were distinctly marked with matching identifiers. The cranium and mandible weights and dimensions were measured and recorded using a pair of digital callipers and set criteria [11] in 3 control and 3 VPA-exposed animals. A total of 18 cranial dimensions were measured in three different parameters, with measurements 1 to 7 quantifying the rostral to caudal lengths, 11 to 17 the lateral dimensions, and 8 to 10 in addition to 18 quantifying the oral cavity dimensions (Figs. 7F, G).

\section{Statistical analysis}

All statistical tested were done using GraphPad Prism 8. Data sets were tested against the normal distribution using the D'Agostino and Pearson normality test. If the normal distribution was met, a one-sided unpaired t-test with Welch's correction was used to assess the differences between the means of VPA-exposed and control rats. A nonparametric Mann-Whitney $U$ test was used under non-normally distributed data conditions. The $\chi^{2}$ test was used to assess difference of the distribution in the frequency of stellate, round, and fusiform neuron cell body shapes between the VPA-exposed and control animals. The difference in VGLUT2 and VGAT terminal densities were assessed between the VPA-exposed and control animals using the 2-tailed unpaired t-test with Welch's correction. Under the condition of non-normally distributed data, the nonparametric 2-tailed Mann-Whitney $U$ test was used. All means were presented as means \pm standard deviation, and $p<0.05$ indicated statistical significance.

\section{RESULTS}

This study focused on four motor nuclei in the brainstem, the TMN, FN, NA and HGN. All four of these nuclei were identifiable in control and VPA-exposed animals and presented no macroscopic structural abnormalities (Fig. 1). There were no differences in the total number of neurons between control and VPA-exposed animals, overall or between right and left sides of the brain (Table 1).

For the TMN, VPA-exposed animals had significantly smaller mean cell body areas compared to controls in the TMN (Figs. 2A, B; 3A; Table 2). When stratified by cell shape, the stellate and round neurons also showed significantly lower mean cell body areas in VPA-exposed animals. There was no significant difference of the cell type distribution in the TMN.

For the FN, VPA animals had significantly smaller mean cell body areas compared to controls (Figs. 2C, D; 3B; Table 2). When stratified by shape, stellate neurons were significantly smaller in VPA-exposed animals (Figs. 2C, D, 3B; Table 2). However, there was no difference for round or fusiform neurons. There was a significant difference in the distribution of these morphologies. In control animals, $80 \%$ of FN neurons were stellate and $20 \%$ round, but in VPA-exposed animals $83 \%$ were stellate, $15 \%$ round and $2 \%$ fusiform. This difference in proportions was significant. 

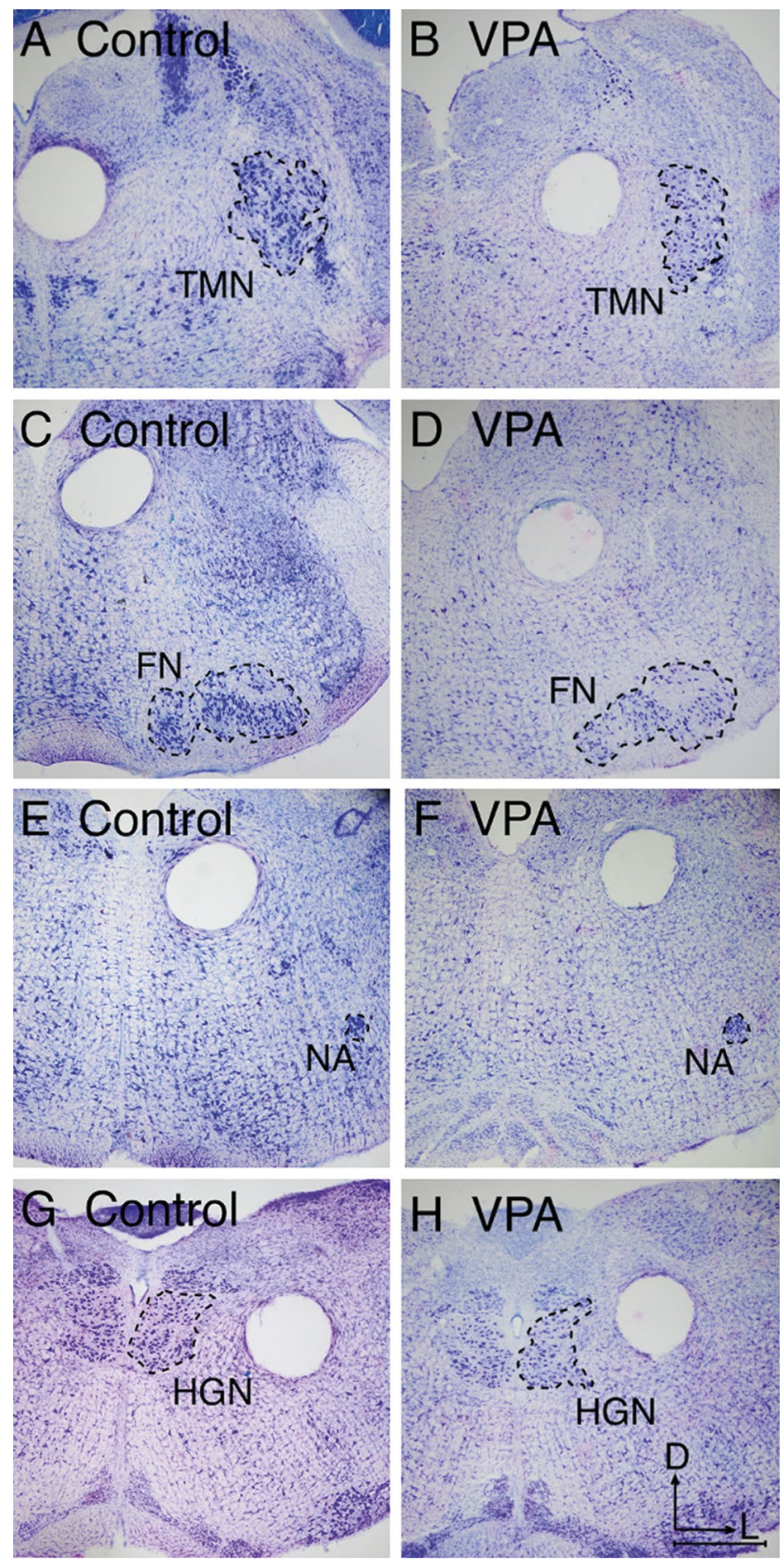

Figure 1. Giemsa-stained sections through the brainstem from control $(\mathbf{A}, \mathbf{C}, \mathbf{E}, \mathbf{G})$ and valproic acid (VPA) (B, D, F, H) animals reveal no change in neuronal number. Images show the trigeminal motor nucleus (TMN) (A, B), facial nucleus (FN) (C, D), nucleus ambiguous (NA) (E, F), and hypoglossal nucleus (HGN) (G, H). The black dashed line indicates the boundaries of each nucleus; D — dorsal; L — lateral. The scale bar equals $500 \mu \mathrm{m}$.

Table 1. Number of neurons

\begin{tabular}{lcccc}
\hline & TMN & FN & NA & HGN \\
\hline Control & $3380 \pm 632.5$ & $6726 \pm 690.3$ & $668.0 \pm 171.1$ & $3530 \pm 389.4$ \\
Valproic acid & $3551 \pm 856.7$ (NS) & $6667 \pm 1034$ (NS) & $616.6 \pm 108.8$ (NS) & $3832 \pm 720.4$ (NS) \\
\hline
\end{tabular}

Data are shown as mean \pm standard deviation; FN — facial nucleus; HGN — hypoglossal nucleus; NA — nucleus ambiguous; NS — not significant; TMN — trigeminal motor nucleus 

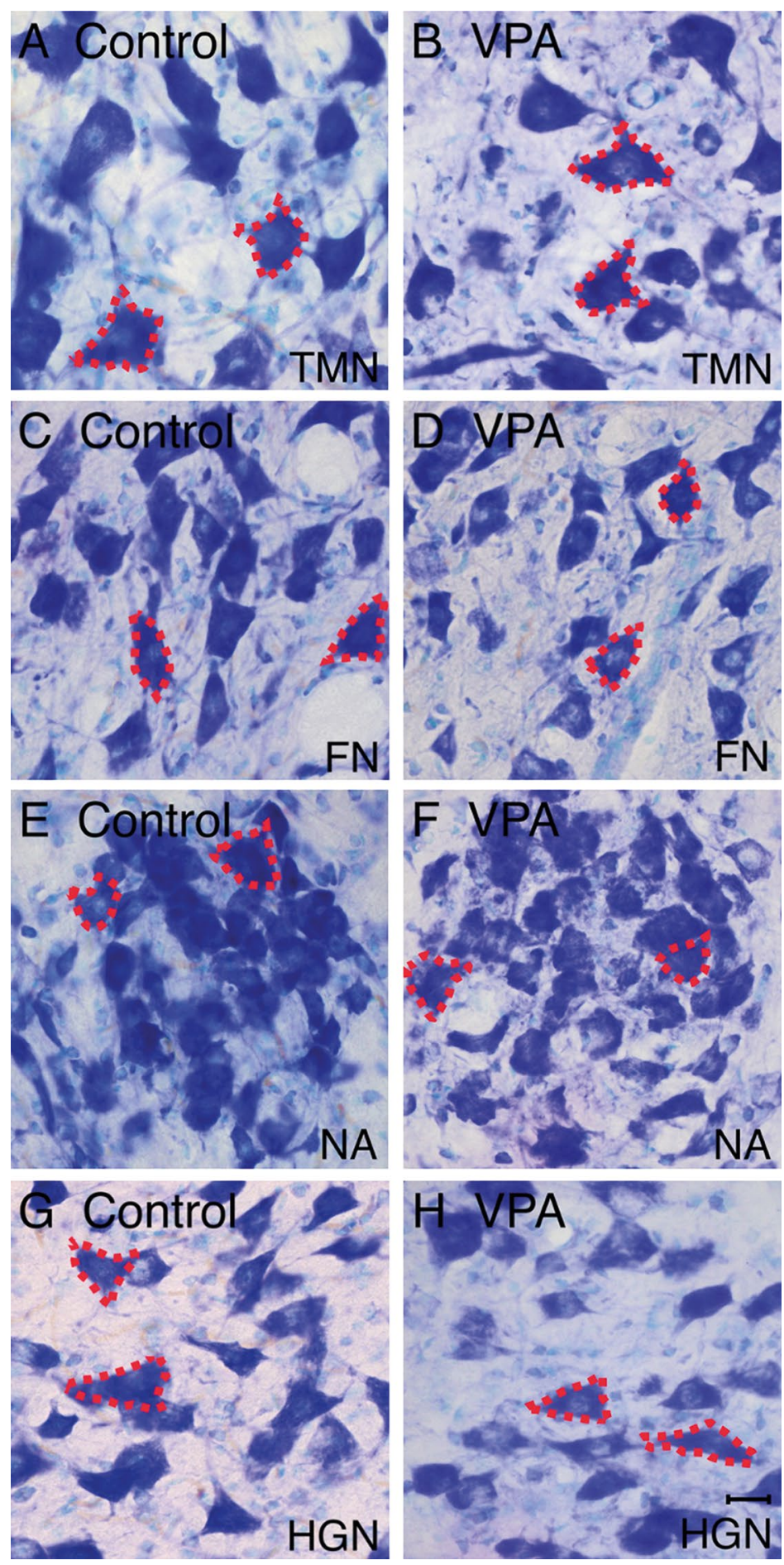

Figure 2. Giemsa-stained sections show lower motor neurons (LMNs) from control (A, C, E, G) and valproic acid (VPA) (B, D, F, H) animals. LMNs are shown from the trigeminal motor nucleus (TMN) (A, B), facial nucleus (FN) (C, D), nucleus ambiguous (NA) (E, F), and hypoglossal nucleus (HGN) (G, H). The red-dashed lines indicate the boundaries of select LMNs. The scale bar represents $20 \mu \mathrm{m}$.

In the NA, VPA-exposed animals had significantly smaller neuronal cell bodies (Figs. 2E, F; 3C; Table 2). This change in cell body size was limited to the stellate neurons; there was no difference in size between round or fusiform neurons. There was no difference in the distribution of neuronal morphologies in the NA.

In the HGN, VPA-exposed animals had significantly smaller neurons (Figs. 2G, H; 3D; Table 2). This 

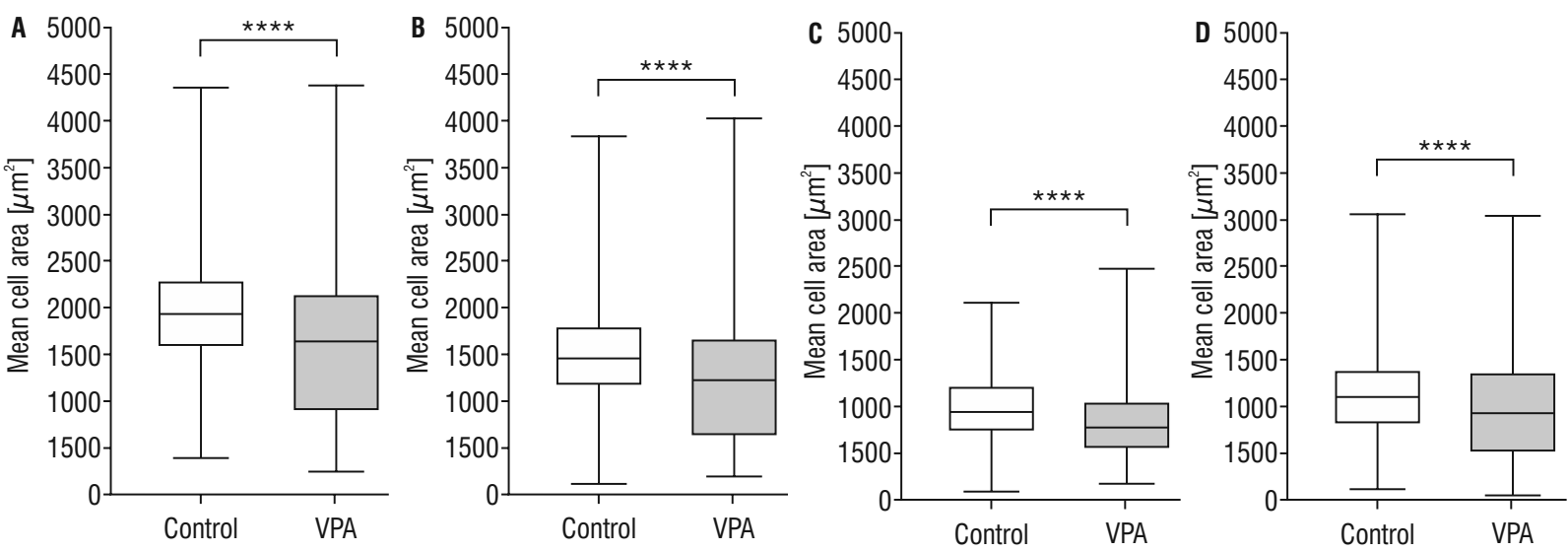

Figure 3. Valproic acid (VPA) exposure resulted in smaller cell bodies. A. Trigeminal motor nucleus; B. Facial nucleus; C. Nucleus ambiguous; D. Hypoglossal nucleus; for each box plot the whiskers represent the maximum and minimum values, the box represents the first and third quartile, and the centre line represents the median; ${ }^{* * *} p<0.0001$.

Table 2. Soma size

\begin{tabular}{cccc}
\hline & Control $\left[\mu \mathrm{m}^{2}\right](\%)$ & VPA $\left[\mu \mathrm{m}^{2}\right](\%)$ & P \\
\hline TMN: & $1925 \pm 636.6$ & $1612 \pm 771.2$ & $<0.0001$ \\
Stellate & $1960 \pm 655.6(79 \%)$ & $1650 \pm 781.5(75 \%)$ & $<0.0001$ \\
Round & $1821 \pm 512.1(20 \%)$ & $1485 \pm 738.0(22 \%)$ & $<0.0001$ \\
Fusiform & $1273 \pm 787.6(1 \%)$ & $1592 \pm 666.6(3 \%)$ & $\mathrm{NS}$ \\
FN: & $1449 \pm 546.1$ & $1234 \pm 655.9$ & $<0.0001$ \\
Stellate & $1458 \pm 546.5(80 \%)$ & $1212 \pm 634.3(83 \%)$ & $<0.0001$ \\
Round & $1427 \pm 541.5(20 \%)$ & $1389 \pm 758.4(15 \%)$ & $\mathrm{NS}$ \\
Fusiform & $980.0 \pm 524.1(1 \%)$ & $986.2 \pm 518.4(2 \%)$ & $\mathrm{NS}$ \\
NA: & $981.2 \pm 368.3$ & $828.6 \pm 399.2$ & $<0.0001$ \\
Stellate & $1024 \pm 407.7(53 \%)$ & $780.9 \pm 421.2(57 \%)$ & $<0.0001$ \\
Round & $939.4 \pm 301.7(46 \%)$ & $903.7 \pm 360.2(40 \%)$ & $\mathrm{NS}$ \\
Fusiform & $801.3 \pm 516.9(2 \%)$ & $749.5 \pm 321.8(3 \%)$ & $\mathrm{NS}$ \\
HGN: & $1074 \pm 477.6$ & $991.9 \pm 571.2$ & $<0.0001$ \\
Stellate & $1064 \pm 511.2(69 \%)$ & $949.1 \pm 561.8(65 \%)$ & $<0.0001$ \\
Round & $1138 \pm 335.5(26 \%)$ & $1114 \pm 584.0(31 \%)$ & $\mathrm{NS}$ \\
Fusiform & $909.0 \pm 553.4(6 \%)$ & $745.2 \pm 439.0(4 \%)$ & $\mathrm{NS}$ \\
\hline
\end{tabular}

Data are shown as mean \pm standard deviation; FN — facial nucleus; HGN — hypoglossal nucleus; NA — nucleus ambiguous; NS — not significant; TMN — trigeminal motor nucleus; VPA — valproic acid

difference in cell body size was restricted to stellate neurons. In control animals, the HGN was composed of $67 \%$ stellate, $26 \%$ round and $7 \%$ fusiform. In VPA-exposed animals the HGN was composed of $65 \%$ stellate, $31 \%$ round and $4 \%$ fusiform and this difference was significant.

\section{VGAT and VGLUT puncta}

In the FN, there was no difference in the density of VGLUT or VGAT-immunoreactive puncta between control and VPA-exposed animals (Fig. 4B; Table 3).
In the TMN, there was no difference in the density of VGAT-IR puncta. However, there were significantly fewer VGLUT-IR puncta (Figs. 4A; 5A, B; Table 3). In the NA, there were significantly fewer VGAT-IR puncta (Fig. 4C; 6A, B), but there was no difference in the number of VGLUT-IR puncta. In the HGN, there were significantly fewer VGAT-IR puncta (Fig. 6C, D) but significantly more VGLUT-IR puncta (Figs. 4D; 5C, D; Table 3).

\section{Skull morphology}

The skulls and mandibles from VPA-exposed animals, on average, weighed more than control animals. However, the differences were not significant (Fig. 7). Measurements of the osteological landmarks shown in Figure 7F-H revealed heavier skulls and mandibles in VPA-exposed animals. VPA-exposed animals had longer rostro-caudal skull and mandible lengths on average (Figs. 7D, measurement $1 ; 7 \mathrm{E}$, measurement 1). However, none of these differences reached statistical significance.

\section{DISCUSSION}

In utero exposure to VPA results in significantly lower body and brain weights after P14 and this might be attributable to oropharyngeal dysfunction. Herein evidence is provided for dysmorphology of oropharyngeal LMNs, excitatory/inhibitory (E/I) imbalance, and possible cranial dysmorphology in animals exposed to VPA. Together, these results support oropharyngeal dysfunction in VPA-exposed animals.

Valproic acid-induced loss of neurons has been found at multiple levels of the auditory brainstem 

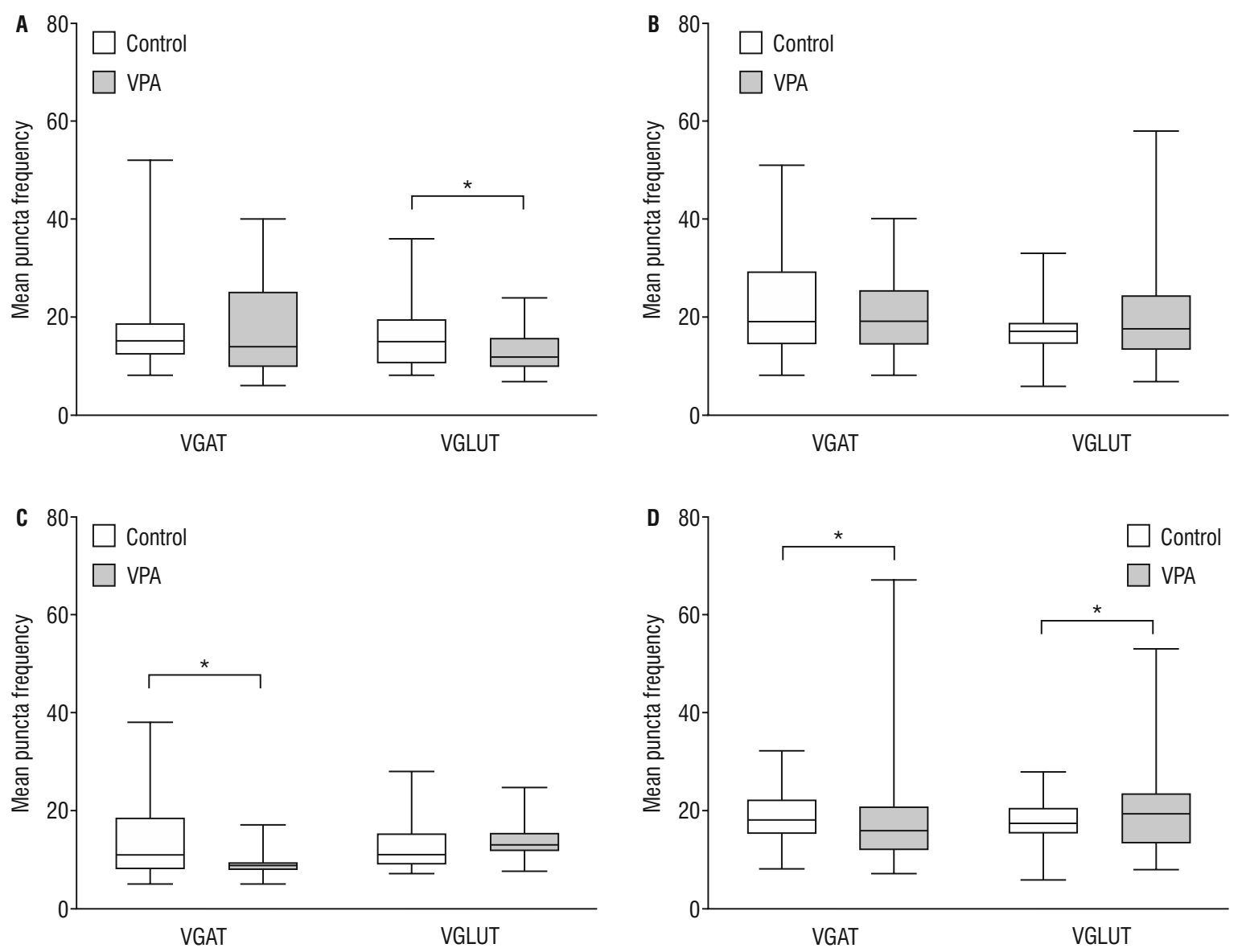

Figure 4. Valproic acid (VPA) exposure led to a significant decrease in VGLUT puncta in the trigeminal motor nucleus (TMN) (A), no change in the facial nucleus (FN) (B), decreased VGAT in the nucleus ambiguous (NA) (C), and significantly fewer VGAT puncta but more VGLUT puncta in the hypoglossal nucleus (HGN) (D). For each box plot the whiskers represent the maximum and minimum values, the box represents the first and third quartile, and the centre line represents the median; ${ }^{*} p<0.05$.

Table 3. VGAT and VGLUT puncta

\begin{tabular}{lccc}
\hline & $\begin{array}{c}\text { Control } \\
\text { [puncta/cell] }\end{array}$ & $\begin{array}{c}\text { VPA } \\
\text { [puncta/cell] }\end{array}$ & P \\
\hline VGAT & & & \\
TMN & $17.21 \pm 8.508$ & $18.13 \pm 10.65$ & NS \\
FN & $21.99 \pm 10.46$ & $20.08 \pm 7.840$ & NS \\
NA & $14.53 \pm 9.690$ & $9.143 \pm 2.535$ & $<0.05$ \\
HGN & $18.72 \pm 5.233$ & $19.81 \pm 13.29$ & $<0.05$ \\
VGLUT & & & \\
TMN & $16.39 \pm 7.274$ & $13.21 \pm 4.329$ & $<0.05$ \\
FN & $17.17 \pm 5.001$ & $19.95 \pm 9.694$ & NS \\
NA & $12.82 \pm 6.277$ & $13.25 \pm 3.173$ & NS \\
HGN & $17.63 \pm 4.533$ & $20.56 \pm 9.136$ & $<0.05$ \\
\hline
\end{tabular}

Data are shown as mean \pm standard deviation; $\mathrm{FN}$ - facial nucleus; $\mathrm{HGN}$ - hypoglossal nucleus; NA — nucleus ambiguous; NS — not significant; TMN — trigeminal motor nucleus; VPA — valproic acid

and thalamus $[22,24,25,41]$. However, there was difference in the number of brainstem LMNs exam- ined in this study. It should be noted that the neuronal estimates provided in this study are consistent with previous reports [15]. The reason VPA exposure does not impact the number of oropharyngeal LMNs is unclear, but it appears that LMNs exhibit some degree of developmental protection. In fact, LMNs are able to reinnervate targets after neonatal injury [18, 33]. Together, these studies provide evidence that brainstem LMNs are less susceptible or able to overcome in utero injury.

Across all oropharyngeal LMNs examined in this study, there were significantly smaller neurons and stellate neurons were most severely affected. In the FN and HGN, there were significant changes in the proportions of the different neuronal morphologies. These changes in cell body size and shape are most likely attributable to smaller, less complex dendritic arbours and/or shorter, less extensive axonal projections. Such changes in the dendritic branching could result in significantly reduced inputs to LMNs. Howev- 

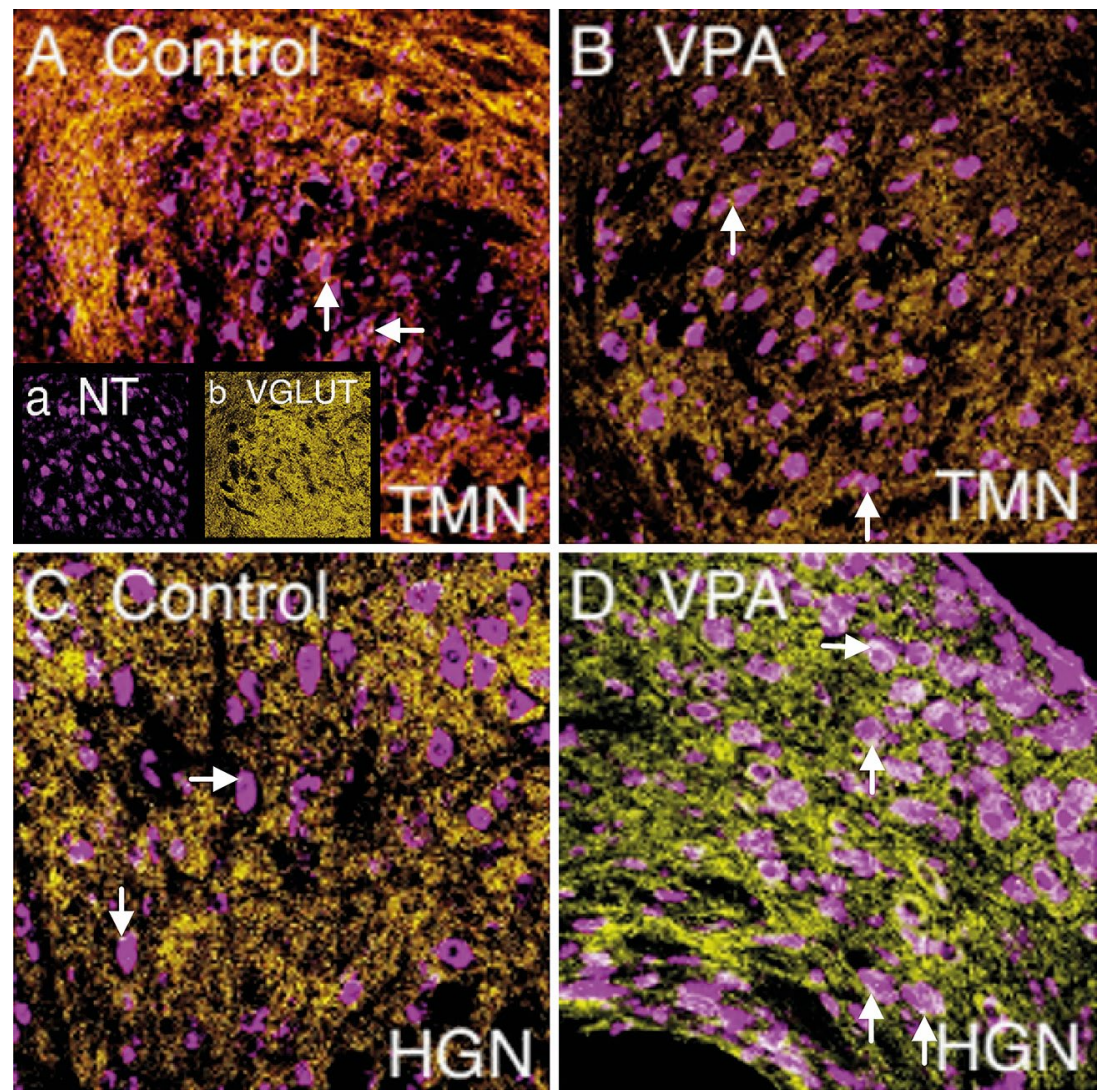

Figure 5. Sections show VGLUT (yellow) and NTR (magenta) labelling and perisomatic VGLUT puncta are indicated by white arrows. Shown in panels A and B are sections through the trigeminal motor nucleus (TMN) (control and valproic acid [VPA], respectively). Shown in panels C and $\mathbf{D}$ are hypoglossal nucleus (HGN) neurons (control and VPA, respectively) NT - neurotrace.
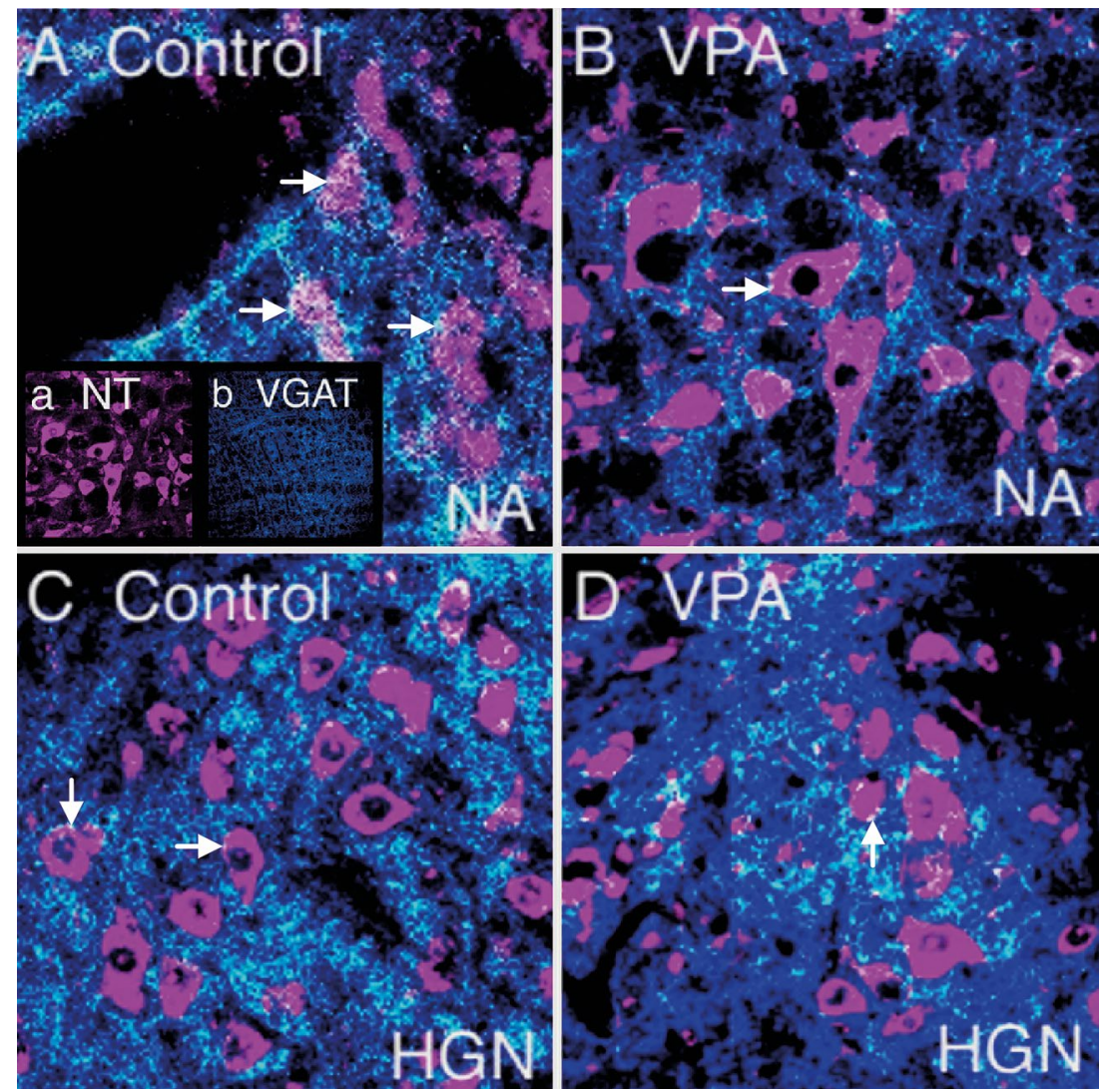

Figure 6. Sections show VGAT (blue) and NTR (magenta) labelling. Perisomatic puncta are indicated by white arrows. Shown in panels $\mathbf{A}$ and $\mathbf{B}$ are sections through the nucleus ambiguous (NA) (control and valproic acid [VPA], respectively). Shown in panels $\mathbf{C}$ and $\mathbf{D}$ are hypoglossal nucleus (HGN) neurons (control and VPA, respectively); NT — neurotrace. 

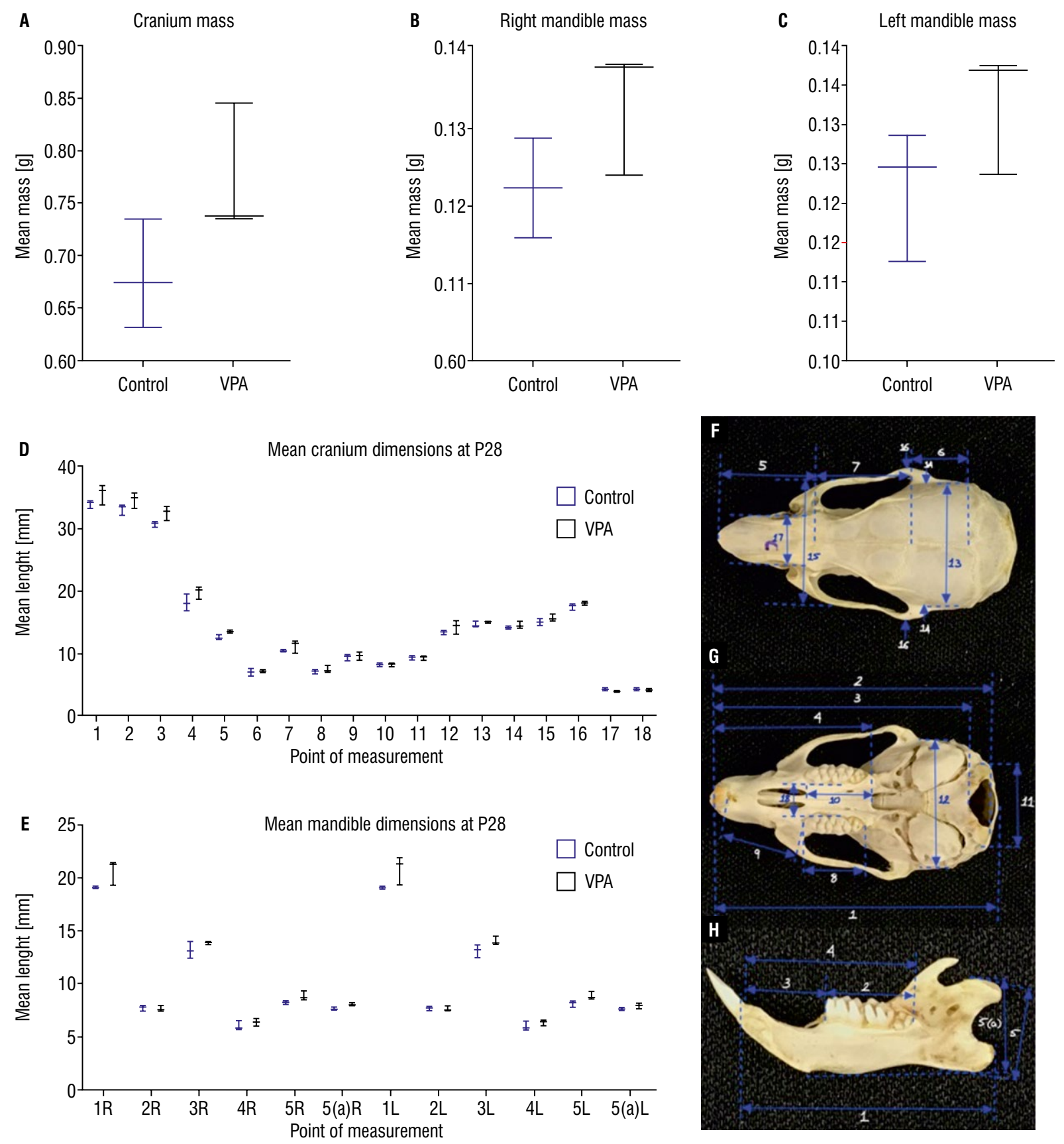

Figure 7. Shown in panel A are overall cranial weights, in panels B and C, right and left mandible masses, respectively. Shown in panels D and $\mathbf{E}$ are comparisons of the skull metrics displayed in panels $\mathbf{F}-\mathbf{H}$. For each plot the whiskers represent the maximum and minimum values; the central horizontal line represents the median; VPA — valproic acid.

er, the size and complexity of LMN dendritic arbours were not examined in the current study.

The number of perisomatic VGLUT and VGAT puncta on oropharyngeal LMNs was also examined as a measure of glutamatergic and GABAergic inputs. The results demonstrate significantly different patterns of E/I inputs to the TMN, NA and HGN. In the TMN, there were fewer VGLUT terminals, consistent with reduced glutamatergic input. The major excitatory input to TMNs is from the mesencephalic nucleus [29]. In the NA, there was reduced VGAT puncta. The majority of inputs to the NA arise from brainstem swallowing centres [19]. In the HGN, there was fewer VGAT puncta but more VGLUT puncta. This finding suggests $\mathrm{E} / \mathrm{l}$ balance may lead to a hyperexcitable environment in the HGN. The sources of these excit- 
atory inputs are the mesencephalic nucleus [40] and reticular formation [34]. The GABAergic projections are also mainly from the reticular formation [34]. A possible limitation of this is that VGLUT and VGAT are surrogates for excitatory and inhibitory synapses and they are not necessarily linked to synapses. Nonetheless, these markers are used to quantify the balance of excitatory and inhibitory inputs.

The impact of VPA on E/I balance on oropharyngeal LMNs does not appear to be uniform across nuclei or systems. In the auditory brainstem, in utero VPA exposure significantly reduces GABAergic neurons and their ascending projections and results in hyperactivity in the midbrain [24, 25, 42]. Regardless, our results support E/l imbalance to brainstem LMNs controlling chewing and swallowing behaviours. How in utero VPA exposure impacts the number of glutamatergic and/or GABAergic synapses at P28 is unclear. However, such lasting effects of VPA on E/l imbalance are most likely due to its role as a histone deacetylase inhibitor (HDAC) $[20,21]$. The changes in $E / /$ balance in the NA and HGN are consistent with increased excitatory input to these neurons. This imbalance might lead to increased muscle tone or spasticity of tongue, pharyngeal, laryngeal and oesophageal muscles and impaired swallowing, reduced caloric intake and poor weight gain. Furthermore, elevated glutamate may result in an excitotoxic environment further impacting LMN function [15]. HDAC inhibition is known to impact proliferation and differentiation of brainstem neurons [16]. Through this mechanism, VPA could impact growth and development of LMN dendritic trees and axonal arbours. In comparing the macroscopic structural differences of the mandibles and cranium of the ASD-rat models to their control counterparts, we saw that the VPA-exposed rats trended towards longer rostro-caudal lengths. However, these differences were not significant. This is counter to the hypothesis that VPA-exposed rats have smaller skulls than the controls. However, examination of muscle bulk, size and innervation may provide further information on control of oropharyngeal function in VPA-exposed animals. Additionally, study of behavioural metrics such as eating and swallowing behaviours may provide further clarification on how these circuits are impacted.

\section{CONCLUSIONS}

In conclusion, this study provides evidence that prenatal exposure to VPA does impact the orofa- cial innervation which leads to possible weaker oropharyngeal musculature, and consequently may elicit oropharyngeal dysfunctions in terms of mastication and dysphagia. The E/l imbalance may also impede the ability to feed through overexcitation leading to spasticity of the muscles, or excitotoxicity in the LMNs leading to oropharyngeal muscle weakness. Characterisation of oropharyngeal dysfunction in animal models will permit a better understanding of feeding disorders in human subjects with ASD and this will lead to development of interventions and therapies to improve quality of life [35].

\section{Conflict of interest: None declared}

\section{REFERENCES}

1. Allen DA. Autistic spectrum disorders: clinical presentation in preschool children. J Child Neurol. 1988; 3 Suppl: S48-S56, doi: 10.1177/0883073888003001s10, indexed in Pubmed: 3198903.

2. Anomal RF, de Villers-Sidani E, Brandão JA, et al. Impaired processing in the primary auditory cortex of an animal model of autism. Front Syst Neurosci. 2015; 9: 158, doi: 10.3389/fnsys.2015.00158, indexed in Pubmed: 26635548.

3. APA.org. [Internet]. What Is Autism Spectrum Disorder? c2018. https://www.psychiatry.org/patients-families/autism/what-is-autism-spectrum-disorder.

4. Baio J, Wiggins L, Christensen DL, et al. Prevalence of autism spectrum disorder among children aged 8 years - autism and developmental disabilities monitoring network, 11 sites, United States, 2014. MMWR Surveill Summ. 2018; 67(6): 1-23, doi: 10.15585/ mmwr.ss6706a1, indexed in Pubmed: 29701730.

5. Bauman ML, Kemper TL. Neuroanatomic observations of the brain in autism: a review and future directions. Int J Dev Neurosci. 2005; 23(2-3): 183-187, doi: 10.1016/j. ijdevneu.2004.09.006, indexed in Pubmed: 15749244.

6. Baxter AJ, Brugha TS, Erskine HE, et al. The epidemiology and global burden of autism spectrum disorders. Psychol Med. 2015; 45(3): 601-613, doi: 10.1017/S003329171400172X, indexed in Pubmed: 25108395.

7. Binkerd PE, Rowland JM, Nau H, et al. Evaluation of valproic acid (VPA) developmental toxicity and pharmacokinetics in Sprague-Dawley rats. Fundam Appl Toxicol. 1988; 11(3): 485-493, doi: 10.1016/0272-0590(88)90112-1, indexed in Pubmed: 3146521.

8. Burdan F, Rozylo-Kalinowska I, Katarzyna Rozylo T, et al. A new rapid radiological procedure for routine teratological use in bone ossification assessment: a supplement for staining methods. Teratology. 2002; 66(6): 315-325, doi: 10.1002/tera.10116, indexed in Pubmed: 12486765.

9. CDC.gov. [Internet]. (2018, 2018-04-26). Data and Statistics | Autism Spectrum Disorder (ASD) | NCBDDD | CDC; c2018. https:// www.cdc.gov/ncbddd/autism/data.html.

10. Christensen J, Grønborg TK, Sørensen MJ, et al. Prenatal valproate exposure and risk of autism spectrum disorders and childhood autism. JAMA. 2013; 309(16): 1696-1703, doi: 10.1001/ jama.2013.2270, indexed in Pubmed: 23613074.

11. Driesch Avd. A guide to the measurement of animal bones from archaeological sites: as developed by the Institut für Palaeoanatomie, Domestikationsforschung und Geschichte der Tiermedizin of the University of Munich. Cambridge, Mass., Peabody Museum of Archaeology and Ethnology, Harvard University; 1976. 
12. Dubiel A, Kulesza RJ. Prenatal valproic acid exposure disrupts tonotopic c-Fos expression in the rat brainstem. Neuroscience. 2015; 311: 349-361, doi: 10.1016/j.neuroscience.2015.10.043, indexed in Pubmed: 26518464.

13. Engineer CT, Centanni TM, Im KW, et al. Speech sound discrimination training improves auditory cortex responses in a rat model of autism. Front Syst Neurosci. 2014; 8: 137, doi: 10.3389/ fnsys.2014.00137, indexed in Pubmed: 25140133.

14. Fombonne E. Epidemiology of pervasive developmental disorders. Pediatr Res. 2009; 65(6): 591-598, doi: 10.1203/PDR. Ob013e31819e7203, indexed in Pubmed: 19218885.

15. Foran L, Kupelian C, Laroia S, et al. Neonatal exposure to monosodium glutamate results in dysmorphology of orofacial lower motor neurons. Folia Morphol. 2017; 76(4): 582-589, doi: 10.5603/ FM.a2017.0052, indexed in Pubmed: 28612917.

16. Fujimura K, Mitsuhashi T, Shibata S, et al. In utero exposure to valproic acid induces neocortical dysgenesis via dysregulation of neural progenitor cell proliferation/ /differentiation. J Neurosci. 2016; 36(42): 10908-10919, doi: 10.1523/JNEUROSCI.0229-16.2016, indexed in Pubmed: 27798144

17. Gandal MJ, Edgar JC, Ehrlichman RS, et al. Validating $\gamma$ oscillations and delayed auditory responses as translational biomarkers of autism. Biol Psychiatry. 2010; 68(12): 1100-1106, doi: 10.1016/j.biopsych.2010.09.031, indexed in Pubmed: 21130222.

18. Harris AJ. Critical periods in the development of motoneurons. Rev Neurol (Paris). 1988; 144(11): 643-647, indexed in Pubmed: 3231955.

19. Holstege G, Graveland G, Bijker-Biemond C, et al. Location of motoneurons innervating soft palate, pharynx and upper esophagus. Anatomical evidence for a possible swallowing center in the pontine reticular formation. An HRP and autoradiographical tracing study. Brain Behav Evol. 1983; 23(1-2): 47-62, doi: 10.1159/000121488, indexed in Pubmed: 6652472.

20. lijima Y, Behr K, lijima T, et al. Distinct defects in synaptic differentiation of neocortical neurons in response to prenatal valproate exposure. Sci Rep. 2016; 6: 27400, doi: 10.1038/srep27400, indexed in Pubmed: 27264355.

21. Kim KiC, Kim P, Go HS, et al. Male-specific alteration in excitatory post-synaptic development and social interaction in pre-natal valproic acid exposure model of autism spectrum disorder. J Neurochem. 2013; 124(6): 832-843, doi: 10.1111/jnc.12147, indexed in Pubmed: 23311691.

22. Lukose R, Schmidt E, Wolski TP, et al. Malformation of the superior olivary complex in an animal model of autism. Brain Res. 2011; 1398: 102-112, doi: 10.1016/j.brainres.2011.05.013, indexed in Pubmed: 21636076.

23. Main SL, Kulesza RJ. Repeated prenatal exposure to valproic acid results in cerebellar hypoplasia and ataxia. Neuroscience. 2017; 340: 34-47, doi: 10.1016/j.neuroscience.2016.10.052, indexed in Pubmed: 27984183.

24. Mansour Y, Ahmed S, Kulesza R. Abnormal morphology and subcortical projections to the medial geniculate in an animal of autism. Exp Brain Res. 2021; 239(2): 381-400, doi: 10.1007/ s00221-020-05982-W, indexed in Pubmed: 33200290.

25. Mansour $Y$, Mangold S, Chosky D, et al. Auditory midbrain hypoplasia and dysmorphology after prenatal valproic acid exposure. Neuroscience. 2019; 396: 79-93, doi: 10.1016/j.neuroscience.2018.11.016, indexed in Pubmed: 30458220.

26. Moore SJ, Turnpenny P, Quinn A, et al. A clinical study of 57 children with fetal anticonvulsant syndromes. J Med Genet. 2000; 37(7): 489-497, doi: 10.1136/jmg.37.7.489, indexed in Pubmed: 10882750.

27. Mychasiuk R, Richards S, Nakahashi A, et al. Effects of rat prenatal exposure to valproic acid on behaviour and neuro-anatomy. Dev
Neurosci. 2012; 34(2-3): 268-276, doi: 10.1159/000341786, indexed in Pubmed: 22890088.

28. Ornoy A. Valproic acid in pregnancy: how much are we endangering the embryo and fetus? Reprod Toxicol. 2009; 28(1): 1-10, doi: 10.1016/j.reprotox.2009.02.014, indexed in Pubmed: 19490988.

29. Pang YW, Ge SN, Nakamura KC, et al. Axon terminals expressing vesicular glutamate transporter VGLUT1 or VGLUT2 within the trigeminal motor nucleus of the rat: origins and distribution patterns. J Comp Neurol. 2009; 512(5): 595-612, doi: 10.1002/ cne.21894, indexed in Pubmed: 19058187.

30. Rodier PM, Ingram JL, Tisdale B, et al. Embryological origin for autism: developmental anomalies of the cranial nerve motor nuclei. J Comp Neurol. 1996; 370(2): 247-261, doi: 10.1002/ (SICI)1096-9861(19960624)370:2<247::AID-CNE8>3.0.CO;2-2, indexed in Pubmed: 8808733.

31. Rueden CT, Schindelin J, Hiner MC, et al. ImageJ2: ImageJ for the next generation of scientific image data. BMC Bioinformatics. 2017; 18(1): 529, doi: 10.1186/s12859-017-1934-z, indexed in Pubmed: 29187165.

32. Schneider T, Roman A, Basta-Kaim A, et al. Gender-specific behavioral and immunological alterations in an animal model of autism induced by prenatal exposure to valproic acid. Psychoneuroendocrinology. 2008; 33(6): 728-740, doi: 10.1016/j.psyneuen.2008.02.011, indexed in Pubmed: 18396377.

33. Sheard $P, M c C a i g ~ C D$, Harris AJ. Critical periods in rat motoneuron development. Dev Biol. 1984; 102(1): 21-31, doi: 10.1016/00121606(84)90171-4, indexed in Pubmed: 6698304.

34. Travers JB, Yoo JE, Chandran R, et al. Neurotransmitter phenotypes of intermediate zone reticular formation projections to the motor trigeminal and hypoglossal nuclei in the rat. J Comp Neurol. 2005; 488(1): 28-47, doi: 10.1002/cne.20604, indexed in Pubmed: 15912497

35. Twachtman-Reilly J, Amaral SC, Zebrowski PP. Addressing feeding disorders in children on the autism spectrum in school-based settings: physiological and behavioral issues. Lang Speech Hear Serv Sch. 2008; 39(2): 261-272, doi: 10.104 4/0161-1461(2008/025), indexed in Pubmed: 18420528.

36. Vorhees CV. Teratogenicity and developmental toxicity of valproic acid in rats. Teratology. 1987; 35(2): 195-202, doi: 10.1002/ tera.1420350205, indexed in Pubmed: 3110992.

37. Werling DM, Geschwind DH. Sex differences in autism spectrum disorders. Curr Opin Neurol. 2013; 26(2): 146-153, doi: 10.1097/ WCO.0b013e32835ee548, indexed in Pubmed: 23406909.

38. WHO.int. [Internet]. Autism spectrum disorders; c2018. https:// www.who.int/news-room/fact-sheets/detail/autism-spectrum-disorders (cited 22 May 2019).

39. Williams G, King J, Cunningham M, et al. Fetal valproate syndrome and autism: additional evidence of an association. Dev Med Child Neurol. 2001; 43(3): 202-206, indexed in Pubmed: 11263692.

40. Zhang J, Luo P, Pendlebury WW. Light and electron microscopic observations of a direct projection from mesencephalic trigeminal nucleus neurons to hypoglossal motoneurons in the rat. Brain Res. 2001; 917(1): 67-80, doi: 10.1016/s0006-8993(01)02911-0, indexed in Pubmed: 11602230.

41. Zimmerman R, Patel R, Smith A, et al. Repeated prenatal exposure to valproic acid results in auditory brainstem hypoplasia and reduced calcium binding protein immunolabeling. Neuroscience. 2018; 377: 53-68, doi: 10.1016/j.neuroscience.2018.02.030, indexed in Pubmed: 29510209.

42. Zimmerman R, Smith A, Fech $\mathrm{T}$, et al. In utero exposure to valproic acid disrupts ascending projections to the central nucleus of the inferior colliculus from the auditory brainstem. Exp Brain Res. 2020; 238(3): 551-563, doi: 10.1007/s00221-020-05729-7, indexed in Pubmed: 31980847 